\title{
Expressão da CASPASE-3 e CD-34 no adenocarcinoma de próstata
}

\section{CASPASE-3 and CD-34 expression in prostate adenocarcinoma}

Vicente Paulo da Motta ${ }^{1}$; Osvaldo Malafaia, ECBC-PR²; Jurandir Marcondes Ribas-Filho, tCbCPR²; Nicolau Gregori Czeczko, TCBC-PR²; Carmen Austrália Paredes Marcondes Ribas²; Ronaldo Máfia Cuenca, TCBC-DF²

\section{R E S U M O}

\begin{abstract}
Objetivo: 1. Avaliar em qual percentual as células tumorais se marcam com caspase-3 e CD-34; 2. quantificá-los nas células tumorais; 3. verificar correlação entre quantificação e grau de malignidade tumoral; 4. correlacioná-los entre si. Métodos: Estudaram-se 38 blocos com adenocarcinoma, classificados por Gleason e marcação imunoistoquímica para caspase-3 e CD-34. As proteínas imunomarcadas foram quantificadas no software Immuno do Sistema Samba 4000 de citofotometria de imagem, pelo índice de marcagem e densidade óptica. Resultados: Imunomarcou-se 25 lâminas para caspase-3 e 34 para CD-34. As quantificações da caspase-3 para o índice de marcagem foram acima de 50 em $76 \%$ e, para densidade óptica, abaixo de 50 para 96\%. Em relação ao CD-34, índice de marcagem foi acima de 50 em 59\% e densidade óptica abaixo de 50 em 56\%. As correlações entre expressões dos marcadores e a gravidade do tumor, assim como entre os marcadores, não evidenciaram significância estatística. Não se mostrou relação de expressão com o score de Gleason. Conclusão: A presença caspase-3 e CD-34 foi de 73,5\% e 100\%, respectivamente; 2. caspase-3 e CD-34 apresentaram alta expressão do índice de marcagem, e baixa para densidade óptica; 3. não houve correlação entre as quantificações com a classificação de Gleason; 4. não houve correlação das expressões dos dois marcadores entre si.
\end{abstract}

Descritores: Caspase 3. Antígenos CD34. Citofotometria. Imunoistoquímica. Adenocarcinoma. Neoplasias da próstata.

\section{INTRODUÇÃO}

A diferenciação entre tumores que evoluirão para doença metastática e aqueles que apresentarão uma evolução lenta e controlável representa um desafio para os pesquisadores ${ }^{1}$.

A patogênese molecular e a progressão do câncer de próstata permanecem pouco conhecidas. Mecanismos genético-moleculares têm sido implicados na tumorigênese prostática e na evolução da doença. Na progressão normal, o ciclo celular apresenta uma sequência de eventos que duplica os componentes celulares e posteriormente ocorre a divisão em duas células. Durante a evolução do câncer, dá-se um processo de multiplicação incontrolável nas células devido às respectivas mutações que ocorreram inicialmente.

Biomarcadores tumorais consistem em indicadores bioquímicos da existência de tumores, incluindo antígenos de superfície celular, proteínas citoplasmáticas, enzimas e hormônios. A morte celular programada, conhecida como apoptose, assim como a angiogênese, apresentam forte correlação com a intensidade da carcinogênese². Os biomarcadores estudados em tais condições tornaramse coadjuvantes importantes na investigação diagnóstica, da progressão do tumor e no tratamento do câncer. Tais marcadores estão incluídos nos fatores prognósticos para carcinoma de próstata na categoria III, segundo o American Pathologist Consensus ${ }^{3}$, descrita como fatores não suficientemente bem estudados para demonstrar seu valor prognóstico.

Marcadores de morte celular programada, tais como a cisteína protease 32, conhecida como caspase-3, responsável pela clivagem proteolítica de várias proteínas, têm sido estudados utilizando-se blocos em parafina confeccionados a partir de diferentes amostras de tumor prostático a fim de quantificar sua expressão ${ }^{4}$.

A perda de controle da apoptose em favor da proliferação celular pode ser responsável pela iniciação e progressão do câncer de próstata'.

Biomarcadores que modulam a angiogênese no câncer de próstata, tais como os antígenos CD-34 expressos nas células endoteliais, também têm sido avaliados através de leitura imunoistoquímica em estudos recentes ${ }^{5}$. A neoformação de vasos dependerá da interação entre as próprias células e com o seu microambiente, incluindo células endoteliais, matriz extracelular e fatores solúveis ${ }^{6}$.

A informática aplicada à área médica possibilitou a análise de imagens de células tumorais através da citofotometria, contribuindo para o diagnóstico de neoplasia7.

A análise computadorizada de imagens histológicas permite quantificar vários marcadores de pro-

Trabalho realizado no Programa de Pós-Graduação em Princípios da Cirurgia e Instituto de Pesquisas Médicas do Hospital Evangélico Universitário de Curitiba/ Faculdade Evangélica do Paraná - Curitiba - PR - Brasil.

1. Mestre, Programa de Pós-Graduação em Princípios de Cirurgia, Faculdade Evangélica de Medicina, Curitiba, Paraná, Brazil; 2. Doutor, Professor Titular, Programa de Pós-Graduação, Faculdade Evangélica de Medicina, Curitiba, Paraná, Brazil. 
teínas, glicoproteínas ou glicoistoquímicos. Dessa forma, dados de microscopia e matemáticos podem ser obtidos a partir da análise; o processamento das imagens da microscopia as transforma em números, possibilitando a elaboração da análise estatística.

O presente estudo sobre o adenocarcinoma de próstata tem como objetivos os seguintes: 1. avaliar o percentual de expressão da caspase-3 e CD-34 nas células tumorais; 2 . quantificar os biomarcadores caspase-3 e CD34 nas células tumorais; 3 . verificar a relação entre os biomarcadores do tumor e sua malignidade; 4. correlacionar os próprios marcadores entre si.

\section{MÉTODOS}

Tecidos de próstata humana foram obtidos de 38 pacientes do Hospital de Brasília, Distrito Federal, Brasil. Todas as amostras de tumores foram identificadas, fixadas em formol, e incluídas em parafina. Além disso, cada corte incluído em parafina foi submetido a nova microtomia com $3 \mu \mathrm{m}$ de espessura. Subsequentemente, as lâminas foram processadas com coloração de hematoxilina-eosina e examinadas independentemente por dois patologistas para um laudo histopatológico e incluídas no estudo. Quatro blocos foram excluídos: a confirmação histopatológica do diagnóstico de adenocarcinoma de próstata não foi efetivada em três deles, e o escore de Gleason não foi determinado para um bloco.

As lâminas foram classificadas segundo o sistema de graduação de Gleason, o qual descreve a infiltração e diferenciação dos tumores, demonstrando que $79,4 \%$ foram considerados malignos, variando de cinco a sete (tabela 1).

O processamento imunoistoquímico seguiu a rotina de preparação de lâminas. As imunoexpressões da caspase-3 e do CD-34 foram obtidas através do complexo streptavidina-biotina-peroxidase. O processamento foi realizado em etapas. Primeiramente, procedeu-se aos cortes histológicos de 3ìm a 5ìm a partir de material incluído em parafina. As seções foram colocadas em lâminas

Tabela 1 - Frequência e percentual do sistema de graduação de Gleason em 34 pacientes apresentando neoplasia prostática.

\begin{tabular}{ccc}
\hline $\begin{array}{l}\text { Sistema de Graduação } \\
\text { de Gleason }\end{array}$ & Número de Casos & $\%$ \\
\hline 2 & 1 & 2,9 \\
3 & 0 & 0 \\
4 & 2 & 5,9 \\
5 & 6 & 17,6 \\
6 & 11 & 32,4 \\
7 & 10 & 29,4 \\
8 & 2 & 5,9 \\
9 & 2 & 5,9 \\
10 & 0 & 0 \\
\hline
\end{tabular}

identificadas. Para a fragmentação da parafina, o material foi incubado a $37^{\circ} \mathrm{C}$ no período da noite. O material foi desparafinizado sob lavagem com xilol e etanol.

Para o anticorpo caspase-3, obteve-se a recuperação do antígeno utilizando-se tampão de solução de citrato, preparado a partir da diluição de ácido cítrico e solução de citrato de sódio em água destilada. Após a secagem, as lâminas foram colocadas em frascos contendo a solução-tampão e foram submetidos a aquecimento. Quando a temperatura atingiu $97^{\circ} \mathrm{C}$, foi cronometrado o tempo de 20 minutos. Os frascos foram então retirados e resfriados. Em seguida, a atividade da peroxidase endógena foi bloqueada através de imersão em peróxido de hidrogênio a $3 \%$.

Com o propósito de manter o $\mathrm{pH}$, as seções foram submetidas a solução-tampão PBS na concentração $0,2 \mathrm{M}$, obtida pela diluição de fosfato de sódio monobásico e fosfato de sódio dibásico, usando Tween 20. Com uma caneta hidrofóbica, traçou-se um círculo em torno do corte histológico. As lâminas foram, então, incubadas com um anticorpo primário contra caspase-3 e/ou CD-34, por um período de 18 horas (da noite para o dia).

A seguir, as lâminas foram lavadas com água destilada e colocadas em solução-tampão PBS. Posteriormente, o anticorpo secundário biotina foi incubado e colocado na solução-tampão PBS. O procedimento de incubação foi realizado com Streptavidina-HRP, seguido por outra lavagem em solução-tampão PBS, quando o cromógeno DAB foi adicionado sobre as lâminas. Subsequentemente, realizou-se a coloração com eosina-hematoxilina. O processo foi finalizado com a preparação e identificação das lâminas.

Anticorpos primários caspase-3 (anticorpo policlonal de coelho anti-CPP32, número de código A3537 Biogen, fração imunoglobulina de antissoro coelho) e CD34 (anticorpo monoclonal de rato, clone QBEnd/10, peso molecular 105-120 kDa, reação de ligação específica para humanos) foram usados no estudo.

Cortes histológicos foram escolhidos para análise imunoistoquímica a partir da coloração da preparação de hematoxilina representando neoplasia e não contendo artefatos técnicos.

A análise de células com imunoexpressão positiva para o anticorpo anti-caspase-3 e para anti-CD34 foi realizada sob microscopia óptica, utilizando-se citofotometria através do Sistema Analisador de Imagem SAMBA 4000, em campos de alta concentração conhecidos como hotspots.

O sistema de análise de imagem era composto por uma câmera de vídeo, dois monitores, impressora, microcomputador e o software específico Immuno® para interpretação e análise de imagens histológicas. O sistema analisador desenvolvido pela Alcatel (Grenoble, França) tem o nome em inglês Microscope Analysis System by Automatic Scanning (SAMBA 4000).

Inicialmente, para que se pudesse utilizar o software, foram feitas a calibragem do sistema e a configuração do padrão. 
As imagens analógicas do microscópio são capturadas através da câmera e enviadas ao computador. O respectivo software analisador permite que o computador forme pontos digitais de imagem (pixels), transformandoos em imagem numérica. Tal processo atribui um valor para cada ponto na imagem. A luz absorvida pelo tecido em cada segmento é quantificada. A quantificação é expressa pela variação na escala de cinza, variando de zero (preto), pontos sem resistência ao feixe de luz, a 255 (branco), apresentando resistência - em outras palavras, onde o tecido é mais denso.

Foi efetuado a varredura do campo visual para localização dos hotspots e o procedimento de leitura foi realizado em barra grega.

Objetivas de 20x foram usadas num número de campos variando de 3 a 10 para cada lâmina, a fim de identificar, no núcleo e no citoplasma, os biomarcadores de apoptose, e em 10 campos por lâmina para identificar, na membrana celular endotelial, os biomarcadores de angiogênese. A superfície total examinada variou de $60.000 \mu \mathrm{m}^{2}$ a $120.000 \mu \mathrm{m}^{2}$. O tempo consumido na leitura de cada lâmina foi de aproximadamente 40 minutos.

Os parâmetros avaliados consistiram em duas variáveis para quantificar a expressão dos marcadores: índice de marcagem e densidade óptica média. $\mathrm{O}$ índice de marcagem descreve o percentual da área marcada. A densidade óptica médica refere-se à intensidade da coloração. Essa variável é expressada em números absolutos.

A interpretação estatística dos dados foi realizada através de análise univariada, calculando-se a expressão, frequência e percentagem dos biomarcadores positivos. No modelo bivariado, foi utilizado o teste exato de Fisher para correlacionar o escore histopatológico de Gleason ( $<7$ ou $=7$ ) com os biomarcadores, os quais também foram subdivididos em dois grupos. O primeiro grupo incluiu valores $<50$, considerados de baixa expressão, e o segundo grupo, de valores $=50$ considerados como alta expressão. Finalmente, considerou-se o grau de associação entre os biomarcadores, por meio do teste de correlação de postos de Spearman, estimando os coeficientes e verificando sua significância.

\section{RESULTADOS}

Foram avaliadas trinta e quatro amostras histopatológicas de pacientes apresentando neoplasia prostática. Todas as amostras foram imunomarcadas para caspase-3 e CD-34, obtendo-se resultados positivos em $73,5 \%$ e $100 \%$, respectivamente $(P=0,002)$.

Nas lâminas marcadas com caspase-3, foi observada uma coloração castanha, tanto para as regiões coradas do núcleo como para as do citoplasma. Foi observada coloração azul nas regiões não coradas (Figura 1).

Nas lâminas marcadas com CD-34, observou-se no endotélio a mesma cor castanha para as regiões coradas e a cor azul para as não coradas (Figura 2).
A quantificação do índice de marcagem e a densidade óptica obtidas para os marcadores são detalhadas nas tabelas 2 e 3 . A caspase- 3 foi considerada em intervalos de 50, em 25 pacientes, e a proteína CD-34 também foi considerada em intervalos de 50, em 34 pacientes.

A tabela 4 demonstra a correlação entre a quantificação do índice de marcagem e da densidade óptica para o marcador caspase-3 e os dois grupos de pacientes definidos por dois graus conforme a classificação de Gleason: $<7$ e $=7$. Nenhuma das correlações foi estatisticamente significativa $(P>0,05)$.

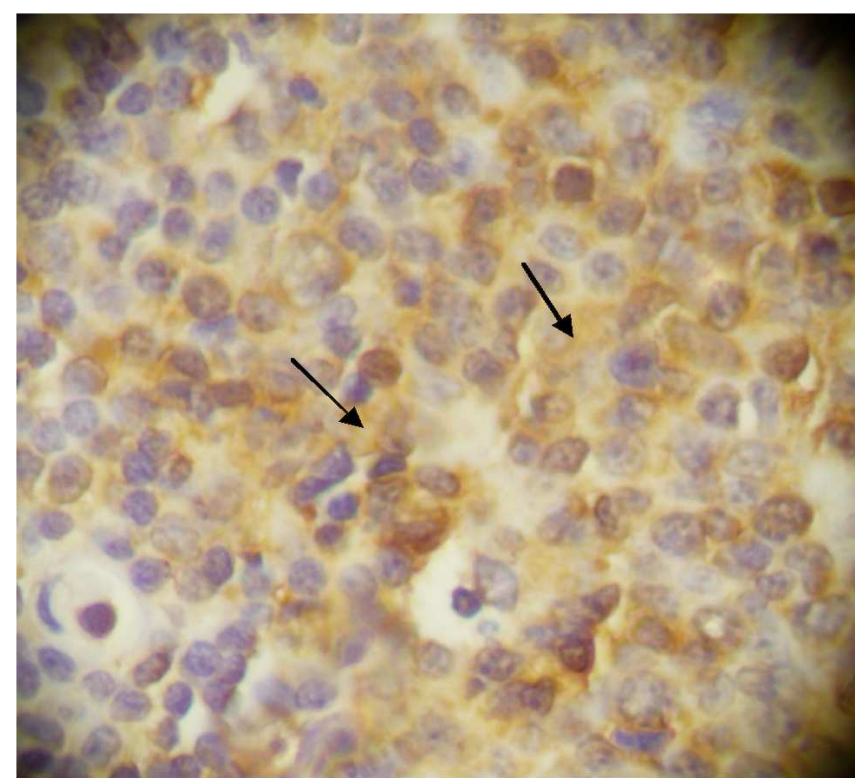

Figura 1 - Microfotografia exibindo o padrão de alta expressão de caspase-3 em adenocarcinoma de próstata grau 6 segundo o escore de Gleason (aumento de 400x). As setas indicam células coradas pelo anticorpo.



Figura 2 - Microfotografia exibindo a alta expressão do padrão de CD-34 em adenocarcinoma prostático grau 6 segundo o escore de Gleason (aumento de 400X). A seta mostra o endotélio imunomarcado. 
A tabela 5 apresenta os dados da correlação entre a quantificação do índice de marcagem e da densidade óptica para o marcador CD-34 e os dois grupos de pacientes definidos pelos respectivos graus de acordo com o escore de Gleason: $<7$ e =7. Nenhuma das correlações foi estatisticamente significativa $(P>0,05)$.

A respeito dos coeficientes de correlação entre caspase-3 e CD-34, o índice de marcagem foi 0,017 $(p=0,936)$ a densidade óptica foi $0,234(p=0,272)$, mostrando que as variáveis não apresentaram significância estatística entre si $(P>0,05)$.

\section{DISCUSSÃO}

Melhorias no diagnóstico precoce do adenocarcinoma de próstata têm visado a novos biomarcadores, devido às limitações no uso do PSA como marcador diagnóstico e prognóstico.

Weidner et al. ${ }^{8}$ concluíram que a descoberta de indicadores prognósticos acurados que tenham correlação com a evolução da doença ajudaria a identificar pacientes que poderiam precisar de terapia agressiva. Atualmente, a origem genética da neoplasia prostática é bem conhecida. Alers et al. ${ }^{9}$ confirmam a possibilidade de documentar alterações cromossômicas associadas com o estadiamento do câncer de próstata.

Gleason ${ }^{10}$ recomenda que seria preferível, em estudos menores de câncer de próstata ou quando o objetivo fosse comparar parâmetros "bons" e "ruins" para tumores, separar os pacientes em dois grupos de acordo com a contagem histológica final em $<7$ ou $=7$. Com base nessa recomendação, o ponto de corte também foi aplicado no presente estudo para comparações na análise estatística.
As células têm a faculdade de passar por uma forma de morte celular programada, conhecida como apoptose, e o crescimento de tumores é determinado por um desequilíbrio entre crescimento e morte celular.

A apoptose consiste numa forma de morte celular programada em organismos pluricelulares, mediada por enzimas proteolíticas conhecias como caspases, responsáveis por iniciar o processo de morte celular pela clivagem de proteínas específicas localizadas no núcleo e no

Tabela 2 - Frequência e percentual referentes ao marcador caspase-3.

\begin{tabular}{lll}
\hline CASPASE-3 & $n$ & $\%$ \\
\hline Índice de marcagem & & \\
$0-50$ & 6 & 24 \\
$51-100$ & 19 & 76 \\
Densidade óptica & & \\
$0-50$ & 24 & 96 \\
$51-100$ & 1 & 4 \\
\hline
\end{tabular}

Tabela 3 - Frequência e percentual referentes ao marcador CD-34.

\begin{tabular}{lcc}
\hline CD-34 & $n$ & $\%$ \\
\hline Índice de marcagem & & \\
$0-50$ & 14 & 41 \\
$51-100$ & 20 & 59 \\
Densidade óptica & & \\
$0-50$ & 19 & 56 \\
$51-100$ & 15 & 44 \\
\hline
\end{tabular}

Correlação entre a quantificação dos biomarcadores segundo o Escore de Gleason

Tabela 4 - Correlação da quantificação entre o marcador caspase-3 e o Escore de Gleason ( $<7$ e =7).

\begin{tabular}{lccc}
\hline CASPASE-3 & GLEASON $<7 n=13$ & GLEASON $=7 n=10$ & VALOR DE $P^{*}$ \\
\hline Indice de marcagem & 3 & 3 & 1,0 \\
$0-50$ & 10 & 7 & \\
$51-100$ & 12 & 10 & 1,0 \\
Densidade óptica & 1 & 0 & \\
$0-50$ & & & \\
$51-100$ & 12 & 0
\end{tabular}

*teste exato de Fisher.

Tabela 5 - Correlação da quantificação entre o marcador CD-34 e o Escore de Gleason (<7 e =7).

\begin{tabular}{|c|c|c|c|}
\hline$C D-34$ & GLEASON $<7 n=20$ & GLEASON $=7 n=12$ & VALOR DE $P *$ \\
\hline \multicolumn{4}{|c|}{ Índice de marcagem } \\
\hline $0-50$ & 10 & 4 & 0,471 \\
\hline $51-100$ & 10 & 8 & \\
\hline \multicolumn{4}{|c|}{ Densidade óptica } \\
\hline $0-50$ & 11 & 8 & 0,71 \\
\hline $51-100$ & 9 & 4 & \\
\hline
\end{tabular}

*teste exato de Fisher. 
citoplasma das células. A família das caspases é composta por 10 tipos de proteínas, sendo a caspase-3 uma das principais executoras do processo celular.

A presença da caspase-3 já foi demonstrada em vários tipos de células ${ }^{11}$. Considera-se que as células epiteliais da próstata estão entre aquelas que apresentam expressão de imunorreatividade positiva para a proteína caspase-3. Na presente pesquisa, verificou-se a presença de caspase-3 em 25 lâminas de um total de 34 (73,5\%). Não obstante isso, há pontos controversos na literatura referente à expressão percentual, com alguns trabalhos demonstrando uma percentagem menor que a apresentada aqui ${ }^{12}$, enquanto outros mostram expressão em todas as lâminas ${ }^{4}$.

A quantificação da caspase-3 foi obtida neste estudo por imagens de computador utilizando o software Immunoß, que expressou numericamente o índice de marcagem e a densidade óptica. Tal método permitiu a exclusão de intervenção subjetiva na análise dos dados. A revisão da literatura mostrou que relatos de quantificação foram obtidos numa análise subjetiva na maioria dos casos ${ }^{4}$. Portanto, no presente estudo, os resultados diferentes observados quando comparados com a literatura podem ser explicados pelos métodos diferentes de análise quantitativa usados na pesquisa recente.

A respeito da correlação entre a quantificação da caspase-3 e a severidade da doença com base no escore de Gleason, a presente pesquisa não mostrou associação significativa. Muitos estudos têm sido elaborados na busca por possíveis correlações, apresentando dados controversos sobre a questão, alguns descrevendo ausência de correlação, enquanto outros demonstram a existência de possível associação ${ }^{4}$. Autores ${ }^{13}$ ofereceram alguma explicação sobre os resultados conflitantes obtidos a partir dos estudos, justificando que as divergências poderiam estar na quantidade de anticorpos usada em cada estudo, sua concentração, sua afinidade ao antígeno, período de incubação, sensibilidade do sistema de detecção, e processo de fixação. Outro ponto a ser considerado consiste no fato de que $79,4 \%$ das amostras analisadas no presente estudo foram classificadas como Gleason 5-7, apresentando comportamento biológico variável.

Bostwick et al. ${ }^{3}$, membros do American College of Pathologists (Colégio Americano de Patologistas) afirmaram durante o Consenso de 1999 sobre Fatores Prognósticos no Câncer de Próstata, a respeito do processo de apoptose, os dados insuficientes na literatura para elaborar recomendações, justificando a necessidade do desenvolvimento de pesquisas sobre o tema.

Folkman ${ }^{14}$ afirmou que a angiogênese, na neoplasia, consiste num processo contínuo e vigoroso observado na cicatrização ou nos processos inflamatórios e, em 1986, relatou que tumores sólidos não eram capazes de crescer além de 1-2 mm sem a formação organizada de vasos. Outros autores demonstraram ${ }^{5,13}$ que a fim de promover o crescimento de tumores, torna-se necessário ter a formação de novos vasos na área do tumor, e que o índice de angiogênese tornar-se-ia um fator prognóstico ${ }^{8}$. Tais afir- mativas justificam a importância da compreensão da angiogênese no câncer prostático, conforme se percebeu na presente pesquisa, que demonstrou a presença do processo de angiogênese em todas as lâminas analisadas.

Bigler et al. ${ }^{15}$ e Brawer et al. ${ }^{16}$ foram os pioneiros em avaliar a densidade de microvasos no tumor de próstata, usando um sistema de análise de imagens computadorizado. Siegal et al. ${ }^{17}$ demonstraram uma densidade mais alta de microvasos no centro do tumor prostático do que na sua periferia, sugerindo que os promotores da angiogênese apresentam uma atividade mais intensa na região central do câncer. O conhecimento da topografia dos microvasos tem suas implicações na quantificação do processo de angiogênese obtida através de amostras de biópsia ${ }^{18}$, diferindo do presente estudo no sentido de que as amostras foram obtidas de prostatectomias radicais.

Existem vários tipos de anticorpos anti-células endoteliais e diferentes maneiras de analisar a densidade dos microvasos. Offersen et al. ${ }^{19}$ examinaram dois anticorpos anti-células endoteliais (anti-CD-34 e anti-fator de Von Willebrand ) e dois métodos diferentes para quantificar a angiogênese no câncer de próstata: densidade de microvasos e Chalkley. Ambos os métodos, quando avaliados utilizando-se o anticorpo anti-CD-34, demonstraram uma acurácia maior para esse marcador, revelando ser o CD-34 mais sensível do que o anti-fator de Von Willebrand como marcador de células tumorais. No presente estudo, a contagem de microvasos basearam-se num sistema de leitura computadorizado, excluindo a contagem subjetiva, melhorando, assim, a credibilidade dos dados.

Correlação entre o CD-34 e o escore de Gleason

A associação entre angiogênese e severidade do tumor já foi investigada por diversos autores 5,13,15

Na presente pesquisa, não foi demonstrada correlação entre a expressão dos antígenos tumorais e seu grau de severidade. Tais resultados são controversos quando comparados com a revisão dos dados da literatura.

Bigler et al. ${ }^{15}$ revelaram maior densidade de microvasos em tumores prostáticos quando comparados com áreas benignas, utilizando o anticorpo anti-fator de Von Willebrand. A contagem de microvasos por imagem usou imagens digitais de um software (Bioscan, Edmonds, WA); um método semelhante ao que foi utilizado no presente estudo. Outros autores ${ }^{8}$ mostraram, através do anticorpo anti-fator de Von Willebrand, que a densidade de microvasos presente em tumores de próstata metastáticos era significativamente maior que em tumores não-metastáticos. Embora obtida em hotspots, a contagem de microvasos foi realizada de forma subjetiva e o método de leitura em barra grega não foi aplicado, ao contrário do que ocorreu no presente estudo. Salomão et al. ${ }^{20}$ observaram correlação positiva entre a infiltração de microvasos e o escore de Gleason em um estudo com 210 pacientes.

Resultados controversos encontrados nesta pesquisa não puderam ser explicados devido à pequena amostra de população investigada. Weidner etal. ${ }^{8}$ também apresentaram uma amostra de população pequena para a interpretação do estudo, composta por apenas 15 pacientes. 
Na análise imunoistoquímica, além do fato de que foi realizada contagem subjetiva, foi usada uma quantificação diferente da expressão de anticorpos e biomarcadores, embora o exame de hotspots tenha sido levado em consideração. Na presente investigação, deve-se considerar também o fato de que $78 \%$ das amostras avaliadas foram consideradas de grau médio para malignidade; em outras palavras, não foram consideradas como estadiamento de tumor completamente "bom" ou "mau", em concordância com a descrição de alguns autores ${ }^{18}$ sobre a não existência de correlação entre a graduação patológica no adenocarcinoma de próstata e os escores de Gleason variando de cinco a sete. Bostwick et al. ${ }^{3}$ afirmam que os dados controversos da literatura são justificados pelos métodos diferentes aplicados e a inconstância na interpretação, determinada pelos observadores, bem como o método de leitura usado nos estudos (contagem geral ou hotspots). Devido à importância da angiogênese na biologia tumoral, os autores recomendam que novos estudos sejam desenvolvidos a fim de estabelecer o modelo de pesquisa e critérios de interpretação adequados .

Na presente pesquisa, o índice de marcagem de marcadores tumorais e a avaliação da densidade óptica não mostraram correlação significativa. Os dados da literatura não estão estabelecidos claramente nesse aspecto, portanto, deve-se considerar a realização de novos estudos a fim de definir uma possível relação.

Afirma-se que no epitélio prostático normal a proliferação celular é equilibrada pelo processo de apoptose $^{12}$. A meta em uma terapia eficaz seria corrigir tal processo de crescimento desequilibrado. Dessa forma, tor- na-se essencial o conhecimento sobre o processo de apoptose e sua intervenção adequada . Autores afirmaram $^{6}$ que o conhecimento preciso do processo de angiogênese tem resultado num grande número de agentes farmacológicos testados em estudos pré-clínicos e, atualmente, em ensaios clínicos. Ainda há que se determinar se a terapia da angiogênese em si é eficaz como monoterapia ou em associação com radioterapia, quimioterapia ou imunoterapia.

Esta pesquisa com método computadorizado de expressão de biomarcadores utilizando o sistema SAMBA 4000 é o primeiro ensaio publicado, de acordo com a revisão da literatura. Outros autores descreveram um procedimento semelhante, embora softwares diferentes tivessem sido aplicados ${ }^{15,16}$. O sistema possibilitou quantificar de maneira fácil e rápida a percentagem de área marcada e sua intensidade cromática, evitando erros subjetivos de leitura.

As conclusões a que se pode chegar são as seguintes: 1. os biomarcadores caspase-3 e CD-34 estavam presentes respectivamente em $73,5 \%$ and $100 \%$ das amostras tumorais; 2 . a caspase- 3 apresentou alta expressão com relação ao índice de marcagem em $76 \%$ das lâminas, demonstrando ao mesmo tempo uma baixa expressão na leitura da densidade óptica em 96\% delas; para a proteína CD-34, seu índice de marcagem foi altamente expressivo em 59\% das lâminas, enquanto que a densidade óptica mostrou baixa expressão em $56 \%$ delas; 3 . não foi demonstrada significância estatística entre a expressão dos biomarcadores e a severidade do tumor segundo o escore de Gleason; 4. não se pôde estabelecer correlação significativa entre os próprios marcadores.

\title{
A B S T R A C T
}

\begin{abstract}
Objectives: 1. To evaluate the percentage of caspase-3 and CD-34 expression on adenocarcinoma; 2. to quantify caspase-3 and CD-34 in tumor cells; 3. to verify the relationship between biomarkers and its malignancy; 4. to correlate biomarkers themselves. Methods: Thirty-eight human malignant prostate specimens, Gleason's score, were immunohistochemically stained for caspase-3 and CD-34 protein. Quantification was done under Samba 4000 Immuno System reading, yielding two variables: label index and optical density. Statistical analyses were based on cross-methods involving univariate and bivariate as well as correlation factors among independent variables. Results: Immunostaining was revealed in 25 plates for caspase-3 and 34 for CD-34. Caspase-3 expression for label index was over 50 in 76\%, while for optical density was below 50 in 96\%. CD-34 expression demonstrated label index over 50 in 59\% and optical density below 50 in 56\%. Correlation among expression and severity did not demonstrate to be statistically significant. There was no correlation between protein expression and Gleason's score. Conclusion: 1. Caspase-3 and CD-34 were present respectively in $73.5 \%$ and $100 \%$ of samples; 2. caspase-3 and CD-34 showed high expression regarding label index and low expression in optical density; 3. there was no statistical significance among expressions and tumor severity according to Gleason's score; 4. no significant correlation could be set between the biomarkers themselves.
\end{abstract}

Key words: Caspase 3. Antigens, CD34. Cytophotometry. Immunohistochemistry. Adenocarcinoma. Prostatic neoplasms.

\section{REFERENCIAS}

1. Tu H, Jacobs SC, Borkowski A, Kyprianou N. Incidence of apoptosis and cell proliferation in prostate cancer: relationship with TGFbeta1 and bcl-2 expression. Int J Cancer. 1996; 69(5):357-63.

2. Matsushima H, Goto T, Hosaka Y, Kitamura T, Kawabe K. Correlation between proliferation, apoptosis, and angiogenesis in prostate carcinoma and their relation to androgen ablation. Cancer. 1999; 85(8):1822-7.
3. Bostwick DG, Grignon DJ, Hammond ME, Amin MB, Cohen M, Crawford D, et al. Prognostic factors in prostate cancer. College of American Pathologists Consensus Statement 1999.Arch Pathol Lab Med. 2000; 124(7):995-1000.

4. Anwar S, Ambros RA, Jennings TA, Ross JS, Beza A, Mian B, et al. Expression of cysteine protease protein 32 in prostatic adenocarcinoma correlates with tumor grade. Arch Pathol Lab Med. 2004; 128(6):649-52.

5. Banerjee AG, Liu J, Yuan Y, Gopalakrishnan VK, Johansson SL, Dinda AK, et al. Expression of biomarkers modulating prostate 
cancer angiogenesis: differential expression of annexin II in prostate carcinomas from India and USA. Mol Cancer. 2003; 2:34.

6. van Moorselaar RJ, Voest EE. Angiogenesis in prostate cancer: its role in disease progression and possible therapeutic approaches. Mol Cell Endocrinol. 2002; 197(1-2):239-50.

7. Goddard JC, Sutton CD, Furness PN, Kockelbergh RC, O'Byrne KJ. A computer image analysis system for microvessel density measurement in solid tumours. Angiogenesis. 2002; 5(1-2):15-20.

8. Weidner N, Carroll PR, Flax J, Blumenfeld W, Folkman J. Tumor angiogenesis correlates with metastasis in invasive prostate carcinoma. Am J Pathol. 1993; 143(2):401-9.

9. Alers JC, Rochat J, Krijtenburg PJ, Hop WC, Kranse R, Rosenberg C, et al. Identification of genetic markers for prostatic cancer progression. Lab Invest. 2000; 80(6):931-42.

10. Gleason DF. Histologic grading of prostate cancer: a perspective. Hum Pathol. 1992; 23(3):273-9.

11. Krajewska M, Wang HG, Krajewski S, Zapata JM, Shabaik A, Gascoyne R, et al. Immunohistochemical analysis of in vivo patterns of expression of CPP32 (Caspase-3), a cell death protease. Cancer Res. 1997; 57(8):1605-13.

12. Amirghofran Z, Monabati A, Gholijani N. Apoptosis in prostate cancer: bax correlation with stage. Int J Urol. 2005; 12(4):340-5.

13. Hall MC, Troncoso P, Pollack A, Zhau HY, Zagars GK, Chung LW, et al. Significance of tumor angiogenesis in clinically localized prostate carcinoma treated with external beam radiotherapy. Urology. 1994; 44(6):869-75.

14. Folkman J. Tumor angiogenesis: therapeutic implications. N Engl J Med. 1971; 285(21):1182-6.

15. Bigler SA, Deering RE, Brawer MK. Comparison of microscopic vascularity in benign and malignant prostate tissue. Hum Pathol. 1993; 24(2):220-6.
16. Brawer MK, Deering RE, Brown M, Preston SD, Bigler SA. Predictors of pathologic stage in prostatic carcinoma. The role of neovascularity. Cancer. 1994; 73(3):678-87.

17. Siegal JA, Yu E, Brawer MK. Topography of neovascularity in human prostate carcinoma. Cancer. 1995; 75(10):2545-51.

18. Silberman MA, Partin AW, Veltri RW, Epstein Jl. Tumor angiogenesis correlates with progression after radical prostatectomy but not with pathologic stage in Gleason sum 5 to 7 adenocarcinoma of the prostate. Cancer. 1997; 79(4):772-9.

19. Offersen BV, Borre M, Sørensen FB, Overgaard J. Comparison of methods of microvascular staining and quantification in prostate carcinoma: relevance to prognosis. APMIS. 2002; 110(2):177-85.

20. Salomão DR, Graham SD, Bostwick DG. Microvascular invasion in prostate cancer correlates with pathologic stage. Arch Pathol Lab Med. 1995; 119(11):1050-4.

Recebido em 10/10/2008

Aceito para publicação em 16/12/2008

Conflito de interesses: Nenhum

Fonte de financiamento: Instituto de Pesquisas Médicas (IPEM) - Hospital Universitário Evangélico de Curitiba (HUEC) / Faculdade Evangélica do Paraná (FEPAR) - Curitiba - PR - Brasil.

\section{Como citar este artigo:}

Motta VP, Malafaia O, Ribas-Filho JM, Czeczko NG, Ribas CAP, Cuenca RM. Expressão da caspase-3 e cd-34 no adenocarcinoma de próstata. Rev Col Bras Cir. [periódico na Internet] 2009; 36(3). Disponível em URL: http://www.scielo.br/rcbc

\section{Endereço para correspondência:}

Vicente Paulo da Motta

E-mail: malafaia@evangelico.org.br 